Method development

\begin{tabular}{|c|c|c|}
\hline Step & Description & Number \& example \\
\hline \multicolumn{3}{|l|}{1 Radiology notes } \\
\hline & $\begin{array}{l}\text { a.Select note titles potentially } \\
\text { relevant to IA }\end{array}$ & a. 35,141 notes titles \\
\hline & $\begin{array}{l}\text { b. Extract notes with titles } \\
\text { potentially related to IA }\end{array}$ & b. $2,926,113$ radiology notes \\
\hline \multicolumn{3}{|c|}{2 Possible meaningful terms } \\
\hline & $\begin{array}{l}\text { a.Compile list of root terms } \\
\text { that may indicate erosion }\end{array}$ & $\begin{array}{l}\text { a. } 11 \text { root terms (i.e. ero*, } \\
\text { pencil*cup, irreg }^{\star} \text { ) }\end{array}$ \\
\hline & $\begin{array}{l}\text { b. Query radiology notes for root } \\
\text { term variations }\end{array}$ & $\begin{array}{l}\text { b. } 1178 \text { variations (i.e. erosion, } \\
\text { erotic, erode) }\end{array}$ \\
\hline & c.Select possible & c. 179 possible terms \\
\hline & meaningful terms & (i.e. erosion, erode) \\
\hline \multicolumn{3}{|l|}{3 Annotation } \\
\hline & $\begin{array}{l}\text { a. Extract snippets^ containing } \\
\text { possible meaningful terms }\end{array}$ & $\begin{array}{l}\text { a. } 5000 \text { snippets from } \\
\text { radiology notes }\end{array}$ \\
\hline & b.Classify snippets according to: & b. 4068 classifications with 1017 \\
\hline & $\begin{array}{l}\text { 1) Meaningful term, 2) Relevance } \\
\text { to joint, 3) Attribution to IA, } \\
\text { 4) Affirmation }\end{array}$ & $\begin{array}{l}\text { snippets (in rounds of } 50-417 \\
\text { snippets for NLP training \& } \\
\text { testing) }\end{array}$ \\
\hline \multicolumn{3}{|c|}{4 Rule development } \\
\hline & $\begin{array}{l}\text { a.Identify meaningful terms } \\
\text { representing erosion }\end{array}$ & $\begin{array}{l}\text { a. } 6 \text { terms (pencil * cup, erosion, } \\
\text { erosive, etc.) }\end{array}$ \\
\hline & $\begin{array}{l}\text { b. Exclude erosive processes } \\
\text { irrelevant to joint(s) }\end{array}$ & $\begin{array}{l}\text { b. } 28 \text { irrelevant processes } \\
\text { (i.e. gastric erosion) }\end{array}$ \\
\hline & $\begin{array}{l}\text { c. Exclude articular erosive } \\
\text { processes not attributed to IA }\end{array}$ & $\begin{array}{l}\text { c. } 5 \text { non-IA processes IA } \\
\text { (i.e. infection) }\end{array}$ \\
\hline & $\begin{array}{l}\text { d. Classify as affirmed/negated } \\
\text { (erosion present/absent) }\end{array}$ & d. 83 affirmation/negation rules \\
\hline 5 NLP training & $\begin{array}{l}\text { Design \& revise NLP model } \\
\text { until accuracy } \geq 90 \%\end{array}$ & $\begin{array}{l}6 \text { rounds, } 817 \text { snippets (AS 417, } \\
\text { RA 200, PsA 200) }\end{array}$ \\
\hline 6 NLP testing & Test NLP model & $\begin{array}{l}200 \text { snippets (AS 100, RA 50, } \\
\text { PsA 50) }\end{array}$ \\
\hline \multicolumn{3}{|l|}{7 Pt classification } \\
\hline & $\begin{array}{l}\text { a. Develop rules for classifying pts } \\
\text { with discordant snippets }\end{array}$ & a. 5 rules developed in 368 pts \\
\hline & $\begin{array}{l}\text { b. Build reference sample (pts } \\
\text { classified as erosive or non- } \\
\text { erosive via chart review) }\end{array}$ & $\begin{array}{l}\text { b. } 30 \text { IA pts (10 AS, } 10 \text { RA, } \\
10 \text { PsA) }\end{array}$ \\
\hline 8 NLP validation & $\begin{array}{l}\text { Validate NLP model in reference } \\
\text { sample at snippet level }\end{array}$ & $\begin{array}{l}149 \text { snippets (29 AS, } 76 \text { RA, } \\
44 \text { PsA) }\end{array}$ \\
\hline 9 Method validation & $\begin{array}{l}\text { Validate methods (NLP+pt } \\
\text { classification) at pt level }\end{array}$ & 30 IA pts (reference sample) \\
\hline
\end{tabular}

Results: In 168,667 veterans with IA, the mean age was $63.1 \& 90.3 \%$ were male. Method development involved radiology note \& erosion term selection, rule development, NLP model building, \& method validation. The NLP model accuracy was $94.6 \%$ at the snippet level $\& 90.0 \%$ at the patient level, for all IA patients.

Accuracy of methods.

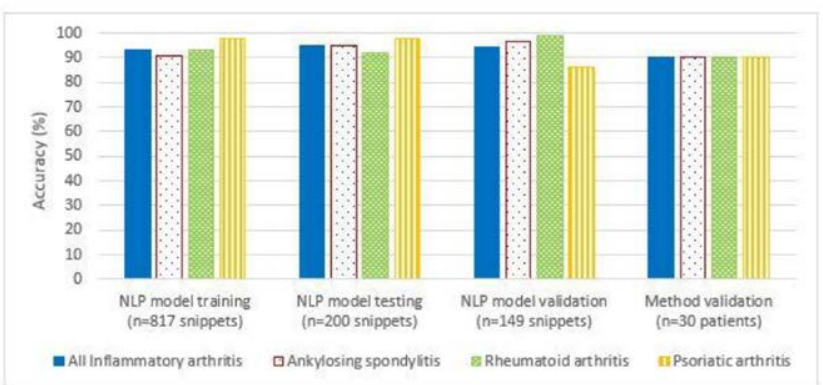

Conclusion: The methods accurately identify erosions from radiology reports of veterans with IA. They may facilitate a broad range of research involving cohort identification \& disease severity stratification

REFERENCES:

[1] Walsh JA, et al. J Rheumatol. 2020;47(1):42-49

Disclosure of Interests: Gopi Penmetsa: None declared, Shaobo Pei: None declared, Brian Sauer Grant/research support from: I have been an investigator on research contracts supported by Abbvie., Jessica A. Walsh Consultant of: AbbVie, Amgen, Janssen, Lilly, Novartis, Pfizer, UCB, Grant/ research support from: AbbVie, Merck, Pfizer, Bingjian Feng Grant/research support from: Bing-Jian Feng reports funding and sponsorship to his institution on his behalf from Pfizer Inc., Regeneron Genetics Center LLC, and Astra Zeneca (UK). The PERCH software, for which Bing-Jian Feng is the inventor, has been non-exclusively licensed to Ambry Genetics for clinical genetic testing services and research., Jodi Walker Shareholder of: Abbvie and mutual funds containing various pharmaceutical companies, Employee of: Abbvie, Kevin Douglas Shareholder of: employed by Abbvie, Employee of: employed by Abbvie, Jerry Clewell Shareholder of: Own Abbvie Shares and mutual funds that hold pharmaceutical and other health care stocks Employee of: I am current Abbvie Inc employee and past employee of Eli Lilly co

DOI: 10.1136/annrheumdis-2021-eular.1794

\section{POS0263 CLINICAL RELEVANCE OF DFS70 ANTIBODIES - A MULTICENTRE STUDY}

A. S. Pinto ${ }^{1}$, J. P. Vilas ${ }^{2}$, F. Cunha Santos ${ }^{1,3}$, M. Cunha ${ }^{4}$, B. Samões ${ }^{5}$, A. Eduarda Porto Raposo ${ }^{6}$, P. Abreu ${ }^{7}$, E. Costa $^{8}$, N. Madeira ${ }^{9}$, J. F. Ferreira ${ }^{1,10}$ C. Vaz ${ }^{1,10}$. ${ }^{1}$ Unidade Local de Saúde da Guarda, Serviço de Reumatologia, Guarda, Portugal; ${ }^{2}$ Baixo Vouga Hospital Centre, Rheumatology Department, Aveiro, Portugal; ${ }^{3}$ University Hospital Centre of Coimbra, Rheumatology Department, Coimbra, Portugal; ${ }^{4}$ Garcia de Orta Hospital, Rheumatology Department, Almada, Portugal; ${ }^{5}$ Vila Nova de Gaia/Espinho Hospital Centre, Rheumatology Department, Vila Nova de Gaia, Portugal; ${ }^{6}$ Trás-Os-Montes e Alto Douro Hospital Centre, Rheumatology Department, Vila Real, Portugal; ${ }^{7}$ Local Health Unit of Castelo Branco, Rheumatology Department, Castelo Branco, Portugal; ${ }^{8}$ Hospital of Braga, Rheumatology Department, Braga, Portugal; ${ }^{9}$ Portuguese Institute of Rheumatology, Rheumatology Department, Lisboa, Portugal; ${ }^{10}$ Beira Interior University, Faculty of Health Sciences, Covilhã, Portugal

Background: Anti-Dense Fine Speckled 70 (DFS70), also known as lens epithelium-derived growth factor (LEDGF) is a common finding when ANA are positive $(1.7 \%$ in the whole population and $4.6 \%$ in the ANA-positive samples). DFS70 antibodies are rare in SARD, especially in the absence of clinical evidence or concomitant anti-extractable nuclear antigen (ENA) antibodies.

Objectives: Our study aimed to understand the meaning of anti-DFS70 antibodies and characterize the clinical and serological features of patients with antiDFS70 positivity.

Methods: We performed a retrospective observational study of consecutive patients followed up at 9 Portuguese Rheumatology Centres observed from January 2018 until April of 2020 with anti-DFS70 antibodies positivity. Descriptive statistics were presented as mean \pm standard deviation if normally distributed or as median and interquartile range if non-normally distributed (continuous variables) or as absolute and relative frequencies (categorical variables). Sensibility and specificity were calculated. Positive and negative predictive values were calculated between patients with and without SARD-specific autoantibodies. Associations between DFS70 with other disease-specific antibody and clinical manifestations were tested using Chi-Square or Fischer's Exact Test, as appropriate.

Results: 120 patients were included, $99(82.5 \%)$ were female with a mean age of $47.8 \pm 18.2$ years. $96.7 \%$ of the patients had ANA titters $\geq 1: 160(32.5 \%$ $1: 160 ; 38.3 \% 1: 320 ; 16.7 \% 1: 640 ; 7.5 \% 1: 1280$ and $1.7 \% 1: 2560)$ and $3.3 \%$ ANA $<1: 160$.

The main clinical reasons for ANA determination was arthralgia (44.2\%), arthritis (11.6\%) and Raynaud Phenomenon (RP) (10\%). The main analytical reason $(7.5 \%)$ was an elevation of inflammatory parameters (C-Reactive Protein (CRP) or Erythrocyte Sedimentation Rate (ESR), leukopenia (3.3\%) and anaemia $(2.5 \%)$. Concerning the immunology: $58.3 \%$ of patients didn't have an associated antibody, $9.2 \%$ had a positive rheumatoid factor, $5.8 \%$ positive ds-DNA, $4.2 \%$ histone and 3.3\% SS-A. 26 patients had more than one associated antibody.

$30(25 \%)$ patients were healthy; $43(35.8 \%)$ patients had Systemic Autoimmune Rheumatic Diseases (SARD) and 47 patients $(39.2 \%)$ had other diseases (nonSARD). Among patients with a SARD, 16 patients presented an isolated positive anti-DFS70 and 27 patients had other antibodies associated. There was found a positive association with non-SARD and arthralgia $(p=0.001)$ and SARD with arthritis $(p<0.001)$. There was an association with SARD and raised inflammatory parameters $(p=0.045)$, but no association was found with anaemia $(p=1.000)$ or leukopenia $(p=0.131)$. Comparative analysis is described in Table 1, with chisquare or Fischer tests, as appropriated.

The sensitivity of isolated DFS70 was $70.1 \%$ and specificity was $62.8 \%$. The positive predictive value was $77.1 \%$ and the negative predictive value was $54.0 \%$. 
Table 1. Comparative analysis using chi-square or Fischer test

\begin{tabular}{lccc}
\hline & SARD & Non-SARD & p-value \\
\hline Arthralgia, $\mathrm{n}$ & 10 & 43 & 0.001 \\
Arthritis, $\mathrm{n}$ & 14 & 0 & $<0.001$ \\
Raynaud Phenomenon, $\mathrm{n}$ & 7 & 5 & 0.114 \\
Raised Inflammation parameters, $\mathrm{n}$ & 6 & 3 & 0.045 \\
Anaemia, $\mathrm{n}$ & 1 & 2 & 1.000 \\
Leukopenia, $\mathrm{n}$ & 3 & 1 & 0.131 \\
\hline
\end{tabular}

Conclusion: We concluded that $64.2 \%$ of patients with positive DFS70 did not present a SARD and if we only consider patients with isolated anti-DFS70, $77.1 \%$ didn't present a SARD. Therefore, in our study, $22.9 \%$ of the patients presented a SARD, which was associated with some clinical features like arthritis or raised inflammatory parameters $(p<0.05)$. Although isolated anti-DFS70 are not specific of a particular condition, our study supports that it can be used as a negative predictor of SARD, if a correlation with clinical and laboratory features is made.

REFERENCES:

[1] Leuchten, N. et al. Performance of Antinuclear Antibodies for Classifying Systemic Lupus Erythematosus: A systematic literature review and meta-regression of diagnostic data. Arthritis Care Res. 70, 428-438 (2018).

[2] Carter JB, Carter S, Saschenbrecker S, Goeckeritz BE (2018) Recognition and relevance of anti-DFS70 autoantibodies in routine antinuclear autoantibodies testing at a community hospital.

Disclosure of Interests: None declared

DOI: 10.1136/annrheumdis-2021-eular.1201

\section{POS0264 THE EMERGING ROLE OF MAGNETIC RESONANCE IMAGING IN INTERSTITIAL LUNG DISEASE IN SYSTEMIC SCLEROSIS: EVIDENCE FOR ULTRA SHORT TE AND COMPRESSED SENSING VIBE ACQUISITIONS AS PROMISING TOOLS FOR THE EVALUATION OF PARENCHYMAL ALTERATIONS}

N. Landini ${ }^{1}$, M. Orlandi ${ }^{2}$, M. Occhipinti ${ }^{3}$, C. Nardi ${ }^{3}$, L. Tofani ${ }^{2}$, S. BellandoRandone $^{2}$, C. Bruni ${ }^{2}$, M. Matucci-Cerinic ${ }^{2}$, G. Morana ${ }^{1}$, S. Colagrande ${ }^{3} .{ }^{1} \mathrm{Ca}^{\prime}$ Foncello General Hospital, Department of Radiology, Treviso, Italy; ${ }^{2}$ University of Florence, Department of Experimental and Clinical Medicine, Florence, Italy; ${ }^{3}$ University of Florence-Azienda Ospedaliero-Universitaria Careggi, Department of Experimental and Clinical Biomedical Sciences,

Florence, Italy

Background: Interstitial lung disease (ILD) is a frequent complication and the major cause of death in Systemic sclerosis (SSc). Computed tomography (CT) is the gold standard imaging technique to assess ILD but is burdened by exposure to ionizing radiations that limits its use for the follow-up. MRI sequences with Ultra Short Echo Time (UTE) are promising for ILD.

Objectives: We tested two MRI sequences, UTE Spiral VIBE and Compressing Sensing (CS) VIBE, in SSc-ILD, in comparison to chest CT.

Methods: SSc patients with suspected-ascertained ILD were evaluated for undergoing CT-MRI examinations in the same day. Two radiologists visually scored the extent of ground glass opacities (GGO), reticulations, honeycombing and consolidations on CT-MRI. The sum of alteration was assumed as ILD extent. A quantitative texture analysis (qCT) was also performed on CT. Cohen's $\mathrm{k}$ was adopted for interreader concordance in ILD detection. MRI sensitivity and specificity in ILD detection were evaluated. Lin's concordance was adopted to compare extent analysis between readers and between CT (visual and qCT analysis) and MRI sequences.

Results: 54 patients performed both CT and MRI. MRI interreader concordance was moderate in ILD detection, while ILD and GGO extent analysis showed good or very good concordance. UTE Spiral VIBE had a sensitivity and specificity in ILD detection of $95.8 \%$ and $77.8 \%$, while alterations extent analysis obtained a very good concordance with CT for ILD and GGO. CS VIBE showed a sensitivity and specificity in ILD detection of $46.7 \%$ and $95.0 \%$, but a slight or fair concordance with CT in all alterations' extent analysis.

Conclusion: MRI UTE Spiral VIBE sequences are helpful in the evaluation of SSc-ILD. Larger cohorts of patients will be needed to confirm that MRI may be useful in clinical practice, reducing the radiological load of chest CT

\section{REFERENCES:}

[1] Romei C, Turturici L, Tavanti L, et al. The use of chest magnetic resonance imaging in interstitial lung disease: a systematic review. Eur Respir Rev. 2018;27(150):180062. Doi:10.1183/16000617.0062-2018

[2] Miller GW, Mugler JP, Sá RC, Altes TA, Prisk GK, Hopkins SR. Advances in functional and structural imaging of the human lung using proton MRI. NMR Biomed. 2014;27(12):1542-1556. doi:10.1002/nbm.3156

[3] Pinal-Fernandez I, Pineda-Sanchez V, Pallisa-Nuñez E, et al. Fast $1.5 \mathrm{~T}$ chest MRI for the assessment of interstitial lung disease extent secondary to systemic sclerosis. Clin Rheumatol. 2016;35(9):2339-2345. doi:10.1007/ s10067-016-3267-0

[4] Ohno $\mathrm{Y}$, Koyama $\mathrm{H}$, Yoshikawa T, et al. Pulmonary high-resolution ultrashort TE MR imaging: Comparison with thin-section standard- and low-dose computed tomography for the assessment of pulmonary parenchyma diseases: Pulmonary MRI with UTE in Pulmonary Disease. J Magn Reson Imaging 2016;43(2):512-532. doi:10.1002/jmri.25008

Disclosure of Interests: None declared

DOI: 10.1136/annrheumdis-2021-eular.3253

\section{POS0265 CLINICAL AND ULTRASONOGRAPHIC ENTHESITIS IN INFLAMMATORY BOWEL DISEASE WITH AND WITHOUT PSORIASIS}

G. Citriniti ${ }^{1}$, A. Soriano ${ }^{2}$, E. Bertolini ${ }^{2}$, G. Sandri ${ }^{3}$, A. Bertani ${ }^{4}$, I. Tinazzi ${ }^{5}$, F. Martinis ${ }^{1}$, N. Girolimetto ${ }^{1}$, C. Salvarani ${ }^{1,3}$, M. Beltrami ${ }^{2}$, P. Macchioni ${ }^{1}$. ${ }^{1}$ AUSL-IRCCS Reggio Emilia, Rheumatology Department, Reggio Emilia, Italy; ${ }^{2}$ AUSL-IRCCS Reggio Emilia, Medicine department, IBD Unit, Reggio Emilia, Italy; ${ }^{3}$ Rheumatology Unit, University of Modena \& Reggio Emilia, Rheumatology Department, Modena, Italy; ${ }^{4}$ University of Modena \& Reggio Emilia, Gastroenterology Department, IBD Unit, Modena, Italy; ${ }^{5}$ Sacro Cuore Don Calabria Hospital, Negrar, Verona, Italy, Rheumatology Unit, Negrar, Italy

Background: Previous studies have reported an association between psoriasis (PsO) and inflammatory bowel disease (IBD). Coexistence of IBD and $\mathrm{PsO}$ has been associated with significant higher prevalence of enthesitis and dactylitis ${ }^{1}$.

Objectives: To compare the prevalence of clinical and ultrasonographic peripheral enthesis abnormalities in a consecutive series of patients with IBD and psoriasis ( $\mathrm{PsO}$ ) as compared to a group of IBD patients without psoriasis (IBD).

Methods: One-hundred seventy-four IBD consecutive patients [36 PsO and 138 IBD, M/F 91/83, mean age $42.6 \pm 14.7$ years, mean disease duration $110 \pm 12.3$ months] entered the study. A complete clinical examination, including rheumatological history, 66/68 peripheral joint count, MASES and LEI scores, BASDAI, and dactylitis count, was performed at study entry. Laboratory test (ESR, CRP, $\mathrm{Hb}$, fecal calprotectin) were collected. Axial or peripheral SpA diagnosis was made using ASAS criteria ${ }^{2}$

US examination was executed by a rheumatologist blind to clinical data, using an Esaote MyLabClass, $18-6 \mathrm{MHz}$ linear multifrequence transducer both in B-mode and PD-mode. The following sites were examined bilaterally: lateral epicondyle of the humerus, distal quadriceps femoris insertion into the patella, inferior pole of the patella, tibial distal insertion of the patellar tendon, calcaneal insertion of the Achilles tendon, and plantar aponeurosis insertion. Knee and ankle joints were evaluated for synovial hypertrophy, PD signal and fluid effusion. Enthesitis was defined according to OMERACT ${ }^{3}$ and scored as $0-36$ for GUESS and 0-136 for MASEI.

Results: PsO patients had later IBD onset (mean age $39 \pm 14.7$ vs $33 \pm 13.2$ in IBD group, $\mathrm{p}=0.02$ ). There weren't observed any significant differences in IBD duration and Crohn/UC prevalence.

No significant difference between the two groups in rheumatological history and clinical examination was detected, except for familiar history of psoriasis (PsO $44 \%$ vs IBD $16 \%, p<0.001)$. Prevalence of $\mathrm{SpA}$ was $33,3 \%$ in $\mathrm{PsO}$ group and $37 \%$ in IBD group $(\mathrm{p}=0.687)$.

146 patients $(83.4 \%)$ showed structural damage at $\geq 1$ enthesis, 44 patients $(25 \%)$ had at least 1 active enthesitis, with no significant difference between the two groups. PsO group showcased a significantly increased prevalence of patients having $\geq 1$ thickened enthesis $(86.1 \%$ vs $63.9 \%, p=0.009)$ and of PD signal at knee examination ( $11.1 \%$ vs $2.2 \%, p=0.034)$. Higher values of GUESS score were observed in PsO $(8.1 \pm 5.1$ vs $5.8 \pm 3.9, p=0.017)$ Enthesis hypoechogenicity was more prevalent in IBD group (27.5\% vs $11.1 \%$, $\mathrm{p}=0.049$ ) 